\title{
Eliciting Requirements for a Robotic Toy for Children with Autism - Results from User Panels ${ }^{1}$
}

\author{
Ben Robins, Nuno Otero, Ester Ferrari, Kerstin Dautenhahn \\ Adaptive Systems Research Group, School of Computer Science, University of Hertfordshire, U.K., \\ e-mail: (B.Robins, N.R.Otero, E.Ferrari, K.Dautenhahn)@herts.ac.uk
}

\begin{abstract}
The work presented in this paper was carried out within the IROMEC project that develops a robotic toy for children. Play has an important role in child development with many potential contributions to therapy, education and enjoyment. The project investigates how robotic toys can become social mediators, encouraging children with disabilities to discover a range of play styles, from solitary to social and cooperative play (with peers, carersiteachers, parents etc). This paper presents design issues for such robotic toys related specifically to children with autism as the end user target group. In or der to understand the play needs of this user group, and to investigate how robotic toys could be used as a play tool to assist in the children's development, a panel of experts (therapists, teachers, parents) was formed and interviewed. Results of the expert panel interviews highlight key points characterizing the play of children with autism, and key points for consideration in the design of future robotic toys.
\end{abstract}

\section{INTRODUCTION}

As early on as infancy, play activity creates and uses auxiliary stimuli and is a crucial part of the child's development [1]. Vygotsky argued that the biological foundation of behaviour is intertwined with the changing social condition, both are inseparable components at each stage of a child's development. Winnicott, too, emphasized the importance of cultural experience in what he called potential space between the individual and the environment, e.g. baby and mother, child and family, individual and society, [2]. Bruner [3] has argued that the motivation for play, and that play itself, is socially constructed. Meanings are learnt in a social way within a particular context $[3,4]$. Contemporary work in activity theory also shows how children's play is socially and culturally constructed [5].

The work presented in this paper is part of our investigation in the IROMEC project (IROMEC - Interactive RObots as MEdiators Companions), a European FP6 project supported by the European Commission. The project recognizes the important role of play in child development and targets children who are prevented from or inhibited in playing, either due to cognitive, developmental, or physical impairment, which affect their playing skills leading to general impairment in their learning potential, cognitive development, and may result in isolation from the social environment. IROMEC investigates how robotic toys can become social mediators encouraging children with disabilities to

\footnotetext{
${ }^{1}$ The work described in this paper was conducted within the EU project "IROMEC" (Interactive Robotic Social Mediators as Companions) and was co-funded by the European Commission in the 6th Framework Programme under contract IST-FP6-045356.
}

discover a range of play styles, from solitary to social and cooperative play, and provide opportunities for learning and enjoyment involving other children as well as carers/teachers or parents who "join in" the game with the robot. This paper specifically address the role of our team within the IROMEC project concerning the development of robotic toy specifically for children with autism. In the remainder of this paper we will focus on this particular end-user target group.

\section{A END-USER TARGET GROUP-CHILDREN WITH AUTISM}

Autism here refers to Autistic Spectrum Disorders, a range of manifestations of a disorder that can occur to different degrees and in a variety of forms [19]. The exact cause or causes of autism is/are still unknown. Autism is a lifelong developmental disability that affects the way a person communicates and relates to people around them. People with autism often have accompanying learning disabilities ${ }^{2}$. The main impairments that are characteristic of people with autism, according to the National Autistic Society [20], are impairments in social interaction, social communication and imagination (referred to by many authors as the triad of impairments, e.g. [21]). This can manifest itself in difficulties in forming social relationships, the inability to understand others' intentions, feelings and mental states, difficulties in understanding gesture and facial expressions, difficulty in understanding metaphors, having a limited range of imaginative activities etc. People with autism usually show little reciprocal use of eye-contact. They also have a tendency toward repetitive behaviour patterns and resistance to any change in routine. In addition some people with autism have hyper-sensitive sensory conditions. Touch can be excruciating, smell can be overpowering, sound, even at an average volume can hurt, and sight can be distorted [22]

As autism can manifest itself to different degrees and in a variety of forms, not only might children in different schools have different needs, but also children in the same school might show completely different patterns of behavior from one to another, and might have different or even some contradictory needs.

\section{PreliminaRY DESIGN CONSIDERATIONS}

An important question when designing robots to assist the

\footnotetext{
${ }^{2}$ For detailed diagnostic criteria the reader is referred to DSM-IV-TR, the Diagnostic and Statistical Manual of Mental Disorders, American Psychiatric Association, (2000) and ICD-10, Classification of Mental and Behavioural Disorders, World Health Organisation, Geneva (1993).
} 
play of children with autism, and thus possibly also to be used in the therapy and education of children with autism, is whether one should use humanoid robots that closely resemble human beings (e.g. possessing a lot of facial features such as eyes, eyebrows, mouth etc.), as suggested e.g. by [6], or rather utilize machine-like, clearly non-humanoid robots, as argued e.g. in [7]. First evidence presented in our previous work in the Aurora project $[8,9]$ clearly supports the case of using simple robots with few features. Results indicated that children with autism showed a preference for interaction with a robot with a plain/robotic appearance over a fully featured human-like "pretty doll" appearance.

An example of a specific design issue that impacts the way children with autism interact with a robots relates to the presence /absence of eyes or eye-like features. Literature suggests that people with autism usually show little reciprocal use of eye-contact and rarely engage in interactive games. Current work, however, shows that some children do engage in eye contact, or are attracted generally by eyes (whether of a real person's eyes or a drawing on toys), while, on the other hand, other children feel threatened by eyes (see section VII below). One of the advantages of using a robotic toy is that once a robot becomes familiar, it might be possible to gradually change the appearance toward a more human-like appearance, which could also assist the children in generalizing experiences from interactions with robots to interactions with people, in line with the analysis presented by Ferrara and Hill in their studies with different toys for children with autism [10]. Indications toward this could be seen in [9] where a case study investigation showed examples where a child with autism directed his eye-gaze towards a robot's face which was only a simple featureless mask with two holes suggesting eyes, clearly attempting to make eye contact with the robot. This raises some questions regarding what level of complexity of facial features is sufficient to trigger a "socially interactive" response in children with autism. Results from our investigation may inform us about robotic design suitable for the purpose of education and therapy of children with autism and help build robots that incrementally guide the children with autism interacting with robots towards more complex and human-like behaviour (see Fig. 1 below).

\section{METHODOLOGICAL FRAMEWORK}

The conceptual and methodological frameworks for the design of interactive artefacts have taken into consideration the close relationship between people in their complex social environments and artefacts: people's actions are intertwined with the artefacts they use. By acting in the world people transform their environment and are influenced by these transformations. Within cognitive science different recent (or re-discovered) theoretical approaches voice this understanding - Situated Action, Activity Theory or Distributed Cognition (a discussion of the pros and cons of each theory is

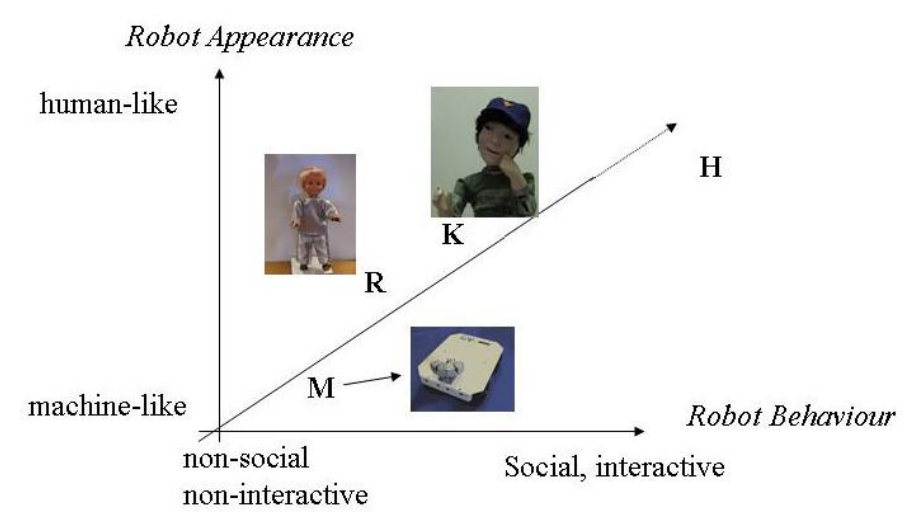

M: mobile machine-like robots

$\mathrm{R}$ : humanoid robot doll Robota

$\mathrm{H}$ : humans

K: humanoid child-sized robot Kaspar

Fig 1 - Appearance and behaviour of different robots that have been used in the Aurora project with the ultimate aim to empower children with autism to deal with the complex nature of human appearance and behaviour (adapted from[11]). Note, the learning trajectory of particular children through this space cannot be expected to be linear.

beyond the scope of this paper, please see $[12,13])$.The influence of these approaches on the methodologies utilized in the design process of interactive products spans from the workplace to home or educational contexts e.g. [14-16].

One important issue regarding the design and evaluation processes of interactive products is the involvement and role of end users in the design teams and project design loop. Some approaches consider users in a more reactive fashion where they are evaluating prototypes or final products. Some other approaches consider the need to include users as full members of the design team. Scaife and Rogers $[17,18]$ proposed the notion of Informant Design, where the central point is to acknowledge the need to consider how different stakeholders with different knowledge/abilities/needs can inform the design at different stages of the development by being prompted by different types of material/artefacts/prototypes. Following a similar approach, our studies regarding the design of a robotic toy for children with autism within the IROMEC project created a framework that includes different phases for the elicitation of requirements and evaluation of prototypes from possible users. In our case, users are not only the children that will play with the toys but also the carers, teachers or parents who might set up the scenario for play and either be part of the play scenario involving the child and the robot, or they may guide/direct the children's interaction with the robot without necessarily being involved in the play directly.

The user panels we conducted are part of the initial design phase of the project in order to find out about the children's interest, in the context of a play scenario, their likes and dislikes, their abilities and needs. Thus, the user panels were set up to elicit initial requirements and give us some understanding of the design space we are facing. Given the nature of the disorder it was not possible to directly include the children in the interviews. Instead we turned to the carers, teachers, parents and expert researchers in order to tap into the extensive knowledge they possess regarding the daily interactions and activities of children with autism. The in- 
volvement of the children will occur in a next stage through exploratory studies where we will test specific design issues in the light of conceptual frameworks or theories regarding autism. Furthermore, involving professional carers in our initial efforts also enabled us to understand their own likes and dislikes and the ways they envision the use of robotic toys in therapy or care.

\section{THE PANEL OF EXPERTS}

Several panels of experts were set by the project's partners in various European countries (i.e. Spain, Italy, The Netherlands, Austria, UK). The panels related to different end-user groups (children with different disabilities; e.g. physical disabilities, cognitive disabilities, autism). The panel described in this paper relates specifically to children with autism.

The UK panel involved professionals from different schools, as well as parents and family members of children with autism. The panel consisted of 7 teachers from 3 different schools, 5 therapists (psychotherapist, speech therapist, play therapist, physiotherapist, occupational therapist) and two parents and family members.

A common methodology was used in all the panels' interviews. This included a short presentation of the project and the aim of the panel meeting, followed by a power point presentation that presented the aims and objectives of the project and gave examples of related previous work. The presentation was followed by a "story telling" session where the members of the panel provided insight into the current play of the children and its characteristics, accompanied with specific examples of the children's play.

The session continued with a "brain storming" discussion amongst the panel members around pre-set questions that aimed to find out which of the play activities / functionality of the robot toys could be of added value for the specific target end-user, which environment this could best take place in, and the critical aspects of the children's behaviour and needs that such a robotic toy could address.

All panel meetings were tape recorded and reports were compiled addressing the key characteristics of the children's play and the key points concerning the design of a robotic toy that might assist the children's play, a summary of which is presented and discussed below.

We organized five different panel meetings in different locations. The key points resulting from these meeting are listed below. Because of the variety of abilities and behaviours of the children, a variety of points are made, some are complementary and some appear contradictory. We have to remember that both the children and their environments are very different.

\section{THE PLAY CONTEXT}

The following are descriptions of the children's play in the context of different environments:

\section{A. Bentfield Primary School - Stansted Mountfitchet, UK}

typically developing pupils. The school also has an Enhanced Provision Unit to cater for nine pupils with various learning difficulties and physical disabilities. These pupils, each accompanied by a Learning Support Assistant, pursue their own unique curriculum and are integrated in the mainstream classes, according to their age group. They participate in any class activity that they are able to.

Play description: according to the teachers, the children here do not have the desire to play and do not get involved in anything "playful" other than their own "obsessive" activity (e.g. playing with running water or moving sand, moving rice grains, moving cars and trains). They can play interactive games with others if they are told to, but they will need to be instructed and supported during the game, otherwise they very quickly return to their "own thing".

\section{B. Middleton School - Ware UK}

Middleton school is a special school for children with moderate learning difficulties, with approximately 92 pupils. The school also has a small base of special classes for children with autism.

Play description: part of the children's routine in this school is starting the day with free un-directed play time. Children are encouraged to do physical games (such as football etc.) outside but also can choose any game indoors. to the incentive is to give them time to do what they choose to do - their own time, their chosen space. It also provides an opportunity for them to engage in their "obsessive" activities (some always play with sand, other always play with trains, other always play with spaceships etc). The children expect it as part of their routine, and they accept when play time is finished and they happily continue with the next, structured, activity of the day. They are more manageable and more focused after that period of play time.

\section{St. Elizabeth School-Much Hadam, UK}

This is specialist residential school that offers education, care and health support for up to 80 young people in the 5-19 age range who have epilepsy and associated disabilities (such as learning difficulties and autism). This includes up to 12 day placements for young people who commute from home

Play description: most of the children will not engage on their own initiative in what we term as "play for pleasure" unless they are told to. Some might, but for a very short time, but if they do so it always involves the same game (e.g. puzzles, jigsaw, etc.).

\section{Meeting with Therapists - University of Hertfordshire, Hatfield, UK}

Play description: the children play solitary and interactive games as well as imaginary and role playing games, however some feel at loss when they appear to want to play with others but don't know how; they don't have the capability to hold the pre-knowledge required (e.g. when acting out a scene from TV programs).

This is a mainstream school with approximately 220 


\section{E. Meeting with parents and family members (of children} with Autism) - Roehampton University, London.

Play description: The children like physical games like chasing, they play solitary games (e.g. intricate arrangements of objects) as well as interactive games. One child likes to initiate interaction with others where there is a less rigid structure. When playing together with a shared object sometimes there seems to be an unspoken negotiation; they can get to an agreement without speaking. Of course there are occasions when a child insists on playing alone.

\section{RESULTS}

The following summarizes the characteristics of the children's play as described by the panel of experts/informants, and what they see as the main aspects to take into consideration when designing a (robotic) toy that could assist the play of children with autism.

\section{A. Key characteristics of the children's play:}

Type of play:

- collaborative - e.g. re-telling a familiar story, in turns, with another person.

- rule-play games - e.g. a board game, they initiate the game and choose a particular person to play with. However some children don't necessarily like board games, or structured rule-games/activities because often the rules are breached and emotionally they can get upset when the rules are broken.

- imaginary play and role-play - some children do play imaginary games and role-play but in a very repetitive manner. Role-play is usually an enactment of a TV program, some children always have to be the same character. Also the episode has to reach the end. Other children when playing role-play with others, are very rigid about their ideas and are unable to accept other people's ideas.

- solitary play is usually very repetitive e.g. always watching the same TV program, printing off the same pictures from the computer or always playing with building bricks.

- $\quad$ solitary imaginary play (e.g. with a Cindy doll)acting out scenes from real life. This could be from TV scenes or what the child has seen, but also could be emotions the child herself was/ had been experiencing. It can be a vehicle to express something she does not yet know how to deal with in real life. Play is not only about interaction - they could play solitary games - and use it to work through things they find difficult in real life. They discover that it is affecting them or others - they learn causality.

- playing alone but in parallel to others - e.g. playing individually with a train set or in the sand, but at the same time being aware of others playing next to them with similar objects.

- some children are at the level of touch /physical sensory level - their play has a "mechanical" nature to it. Although others participate in instructed interactive games, this occurs in very "mechanical" ways.

\section{Movement:}

- the child's own movement - running around etc., or watching something moving on its own, against the non-moving environment (e.g. car racing on tracks). Objects that move around can become the subject of joint attention.

- some children are attracted by movement of objects and enjoy the anticipation of an event at the end of the movement (e.g. a rolling ball falling off a table, a bell, sound of a wind-up clock etc).

- sensory reward - they would not want to participate in a game unless there is a sensory reward element, this reward could be sound, light, movement preferences are different from child to child.

Imitation:

- some children might respond positively when being imitated. Imitation can then be developed into a turn-taking activity. It also might promote taking initiative, e.g. one child was thrilled when people copied him. He took the initiative and was excited by the fact that he was in control - people copied him and repeated what he did.

- however, some children can get really irritated when being imitated.

- interactors - they often seem to play better with an adult than with another child (they don't appear to take any notice of another child in the vicinity).

\section{B. Key points concerning the design of a robotic toy:}

Familiarity:

- a toy that is unfamiliar can be unsettling - so it needs to have built-in familiarity (familiar aspects in looks, sensory output, behaviour etc. ), e.g. if it is a doll then it needs a familiar set of clothes that can be taken on and off.

- on the one hand the robot needs to show some structured behavior so the children know what to expect, but the behaviour should also evolve continually, and thus can help to sustain the children's attention after the novelty wears off.

Choice and control:

- choice - it is VERY important that the child is able to make choices. The robotic toy should have a range of features that are familiar to the specific child (e.g. safe objects/pieces of music/colour of lights) - to let the child choose. Some children prefer a toy that can produce sounds and/or lights etc, so that children can not only watch it, but are actively involved in 'making the interaction happen', exploring the toy etc.

- the robot could be covered by cloth or other material in order to provide more sensory experience.

- the robot could be adapted to a particular set (a living room for example) that provides a context that also can be explored at home. 

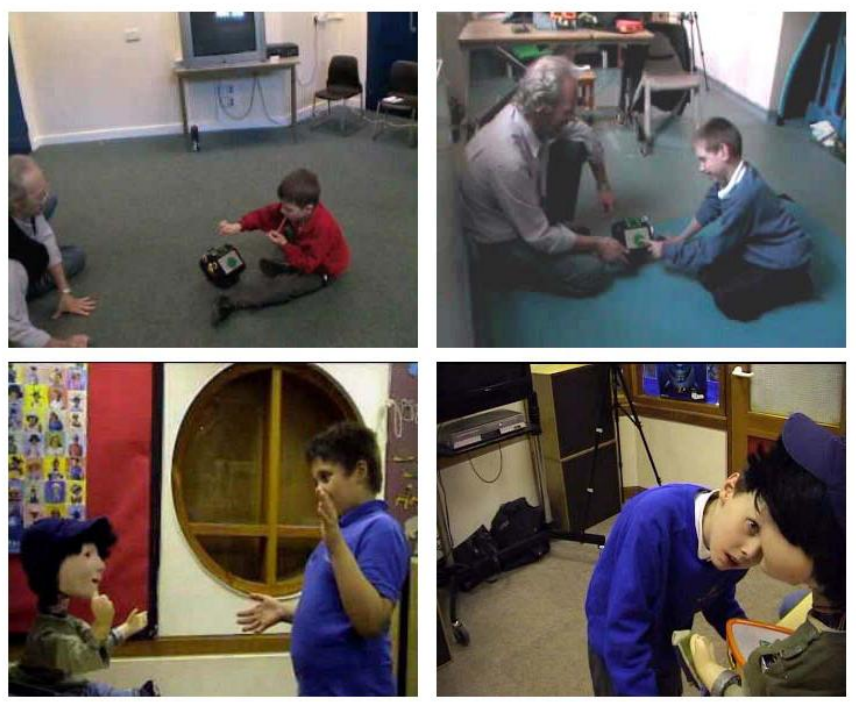

Fig 2 - characteristics of the children's play as described by the panel of experts are being tested in experimental investigations.

- control buttons are required - simple controls on the object itself (as an alternative to remote control) pushing the button for example - which results in the movement /reaction of the toy and it also gives the child the control over the toy's behaviour.

Complexity and Modularity:

- complexity - it needs to be modular - different children would want different levels of technology (e.g. light and sounds) - for some children something very simple with a very limited level of technology might feel quite good; while others would need more complexity to sustain their interest. It needs to be modular and adaptable according to each child's particular preference and at the same time it needs to be adjustable as some children are more sensitive than others. For example some children like music very much - however a lot of the music on children's toys is not necessarily interesting to the children; it needs to be modular and adjustable to the specific child-one child may like Mozart, while another likes children's rhymes etc.

- it needs to be interactive, but starting with very simple interactions and gradually getting more complex - e.g. like pressing buttons that cause the robot to move, or change lights or both.

Appearance:

- $\quad$ some children like the tactile quality of fur; others like the sense of hard plastic

- $\quad$ some children are freaked by big eyes - they will scratch them; others like to see eyes.

- the robot should not be too human like. However having "eyes" could be useful for some children and might encourage interaction (similar to the eyes, for example, on "Thomas the Tank Engine" etc). This needs to be a modular feature as for some children it is painful to look at eyes. Possibly having a symbolic/ "mechanical" face (e.g. like a matchstick person face or a "Smiley" face with dots and lines).

- the toy should not be in a human form but have more "machine-like characteristics" e.g., a rigid toy that produces a sequence of actions.

- appearance - a two dimensional type of an object like cartoons - simple, without too many details.

Behaviour:

- "trigger action" - each child has a different "action" that stimulates him/her (a "trigger action"). In order to motivate the child, the robots must have something similar that will be familiar to the child. Because each child with autism might have different "trigger actions", the robots need to be modular enough to offer different "trigger actions" as appropriate to each child.

- physical manipulation of the object needs to be encouraged. The child needs to be able to manipulate it. The robot's behavior needs to depend on the action of the child. At the same time it is hoped that the robot will encourage the children to move and maybe to stretch different body parts.

\section{Environment and context:}

- the environment should allow the children to interact and make mistakes and still be supportive. It is important that they are received sympathetically even when they don't get it right.

- companionship - such as a partner to play with the child - responsive to the child's movement, receptive and responsive to the child's actions.

\section{DISCUSSION OF RESULTS}

The user panels clearly highlight the fact that the robotic toys may be used in different contexts (at home, at different schools), by diverse children who show strong likes and dislikes as well as a wide range of abilities and needs.

In summary, there is a need to consider the following when designing a robotic toy for children with autism:

- the different types of play according to the specific child's abilities

- the different physical settings that can influence the type of play and interactions (for example, what kinds of movement are possible in a specific physical setting?)

- the complex intertwining of appearance and its influence on the interaction

The results from the user panels present the following robot design challenges:

- How to accommodate the needs of different children in a certain setting (in a particular school)?

- How to accommodate different settings (different schools, home etc)?

- How to accommodate the progression of the child according to therapeutic/educational goals?

- How can the carers of the children adjust the robots' characteristics to the needs they envision?

As stated above, autism has a wide range of manifesta- 
tions where children might display completely different patterns of behaviour from one to another (a variety interaction styles can be seen in Fig 2). The results discussed in section IV above show that children with autism might have different or even contradictory needs. The challenge in providing a high degree of flexibility in an assistive tool to answer these needs, implies that there is a strong requirement for a built-in modularity that could be accessed by the users, and to their choosing.

$\mathrm{Also}$, an important issue to consider in future work concerns the interface that the robot provides to the children (interaction modalities etc.), but, equally important, the interface to carers/teachers/parents who may want to change settings and tune the robot's behaviour.

\section{Conclusion}

Interaction with the environment in play provides stimuli that influence and control the behaviour of the child and is crucial to child development [23]. Here, the interaction between the child and the environment is based on reciprocal stimulation that creates transitions of change and modification. This leads to refinement in the nature of the child's behaviour, which also becomes more orderly. This sequence of actions and reinforcements becomes structured and predictable, and could enhance the quality of the child's behaviour and may affect the speed with which he/she develops. This dyadic model of interaction with the environment could be implemented in robotic systems that can be used with autistic children to provide stimuli and reinforcement in a controlled manner (a gradual increase in complexity) helping the child learn basic social behaviour skills.

Being a programmable system, a robot can provide various stimuli that could promote the child to interact with it in different ways. The ability to modify the response of the robot according to the way the child interacts, and to repeat this modified response, can make the cycle of actions and reinforcement orderly and predictable. Robotic systems could have both, a built-in modularity to accommodate different needs of different children as well as a built-in capability to gradually increase the complexity of the interaction thus providing more complex stimuli that may promote further learning (e.g. simple imitation games might become more complex turn-taking activities).

\section{REFERENCES}

[1] L.S. Vygotsky, Mind in Society, Harvard University Press: Cambridge MA, 1978
[2] D.W. Winnicott, Playing and Reality, Penguin Books Ltd: Middlesex UK, 1971.

[3] J.S. Bruner, Acts of meaning, Harvard University Press: Cambridge MA, 1990.

[4] S. Powell, Helping Children with Autism to Learn, David Fulton Publishers: London UK, 2000.

[5] P. Hakkarainen, "Play and Motivation," in Perspectives on Activity Theory, Y. EngestrÖm, R. Meittinen, and R. Punamaki, Cambridge University Press Eds.: Cambridge, UK, 2003.

[6] C. Breazeal and A. Foerst, "Schmoozing with robots: Exploring the boundary of the original wireless network, " in Proceedings of the CT1999, Third International Cognitive Technology conference: Networking Minds, 1999, pp. 375-390.

[7] K. Dautenhahn, "Robots as social actors: Aurora and the case of autism," in Proceedings of the Third International Cognitive Technology Conference CT99, August, San-Francisco, 1999.

[8] AURORA, URL: http://www.aurora-project.com/ last accessed 06/03/06, 2007.

[9] B. Robins, K. Dautenhahn, and J. Dubowsky, "Does appearance matter in the interaction of children with autism with a humanoid robot?, "Interaction Studies, vol. 7:3, 2006, 479-512.

[10] C. Ferrara and S. D. Hill, "The responsiveness of autistic children to the predictability of social and non-social toys," Autism and Developmental Disorders, vol. 10, 1980, 51-57.

[11] K. Dautenhahn, "Design Spaces and Niche Spaces of Believable Social Robots," in Proceedings of the IEEE Int. Workshop on Robot and Human Interactive Communication ROMAN 2002, 25-27 September, Berlin, Germany, 2002, 192-197.

[12] B. Nardi, Context and Consciousness: Activity Theory and Human-Computer Interaction, MIT Press: Cambridge, MA, 1996.

[13] G. Salomon, (Ed.) Distributed Cognitions: Psychological and educational considerations. Cambridge University Press: Cambridge, 1993.

[14] J.M. Carrol, "Human-computer interaction: psychology as a science of design, "International Journal of Human-Computer Studies, vol. $46,1997,501-522$.

[15] G. Olson and J. Olson, "Human-Computer Interaction: Psychological Aspects of the Human Use of Computing, "Annual Review of Psychology, vol. 54, 2003, 491-516.

[16] Y. Rogers, "New Theoretical Approaches to HCI, "ARIST: Annual Review of Inforation Science and Technology, vol. 38, 87-143, 2004

[17] M. Scaife and Y. Rogers, "Kids as Informants: Telling Us What We Didn't Know or Confirming What We Knew Already? " in The Design of Children's Technology, A. Druin, Ed. Morgan Kaufmann Publishers: San Francisco, 1999, 27-50.

[18] M. Scaife, Y. Rogers, F. Aldrich, and M. Davies, "Designing for or designing with? Informant design for interactive learning environments," in Proceedings of the CHI'97, New York, 1997.

[19] R. Jordan, Autistic Spectrum Disorders - An Introductory Handbook for Practitioners, David Fulton Publishers: London, 1999.

[20] NAS, "National Autistic Society UK, url: http://www.nas.org.uk, last accessed 07/01/07," 2007.

[21] L. Wing, The Autistic Spectrum. London: Constable Press, 1996.

[22] G. Gillingham, Autism: Handle with Care. Understanding and Managing Behaviour of Children and Adults with Autism, Future Education Inc: Arlington TX, 1995.

[23] S. Cohen, Social and personality development in childhood, Macmillan Publishing Company: NewYork and London, 1976. 